\title{
Why are the magnetic field directions measured by Voyager 1 on both sides of the heliopause so similar?
}

\author{
J. Grygorczuk \\ Space Research Centre, Warsaw, Poland \\ jolagry@cbk.waw.pl \\ A. Czechowski and S. Grzedzielski \\ Space Research Centre, Warsaw, Poland
}

Received __; accepted _ 


\begin{abstract}
The solar wind carves in the interstellar plasma a cavity bounded by a surface, called the heliopause (HP), that separates the plasma and magnetic field of solar origin from the interstellar ones. It is now generally accepted that in August 2012 Voyager 1 (V1) crossed that boundary. Unexpectedly, the magnetic fields on both its sides, although theoretically independent of each other, were found to be similar in direction. This delayed the identification of the boundary as the heliopause and led to many alternative explanations. Here we show that the Voyager 1 observations can be readily explained and, after the Interstellar Boundary Explorer (IBEX) discovery of the ribbon, could even have been predicted. Our explanation relies on the fact that the Voyager 1 and the undisturbed interstellar field directions (which we assume to be given by the $I B E X$ ribbon center $(\mathrm{RC})$ ) share the same heliolatitude $\left(\sim 34.5^{\circ}\right)$ and are not far separated in longitude (difference $\sim 27^{\circ}$ ). Our result confirms that Voyager 1 has indeed crossed the heliopause and offers the first independent confirmation that the IBEX ribbon center is in fact the direction of the undisturbed interstellar magnetic field. For Voyager 2 we predict that the difference between the inner and the outer magnetic field directions at the heliopause will be significantly larger than the one observed by Voyager $1\left(\sim 30^{\circ}\right.$ instead of $\left.\sim 20^{\circ}\right)$, and that the outer field direction will be close to the RC.
\end{abstract}

Subject headings: ISM: magnetic fields — Sun: heliosphere 


\section{Introduction}

In August 2012, V1 crossed the HP and entered the local interstellar medium (LISM) (Burlaga, Ness \& Stone 2013; Stone et al. 2013). Contrary to expectations, the change in the magnetic field direction across the HP was small (Burlaga, Ness \& Stone 2013) $\left(\sim 20^{\circ}\right)$. As a result, the identification of the boundary as the HP remained in doubt. This led to speculations about the existence of an unexpected transition layer at the HP that allows enhanced exchange of energetic particles with the interstellar medium while conserving magnetic field characteristics typical for the heliosheath (Fisk \& Gloeckler 2013; Florinski 2013; McComas \& Schwadron 2012; Stone et al. 2013; Schwadron \& McComas 2013). Only detection of electron plasma oscillations which allowed to evaluate the local plasma density at V1 in 2013, with values characteristic of the LISM, provided a strong evidence that the spacecraft has already crossed the heliopause (Gurnett et al. 2013). However it is still not understood why the magnetic field direction observed beyond the HP is so close to the field measured in the inner heliosheath, and attempts are still being made to answer this question

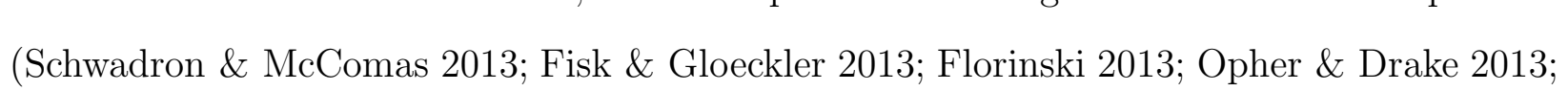
Borovikov \& Pogorelov 2014).

In this letter we show how this observation can be explained in a simple and natural way if the undisturbed interstellar magnetic field (ISMF) far from the heliosphere points to the IBEX RC (Funsten et al. 2009). The ribbon is an almost circular arc in the sky of enhanced energetic neutral atoms emission discovered by the Interstellar Boundary Explorer (IBEX) (McComas et al. 2009; Funsten et al. 2009). The RC is widely interpreted as the direction of the undisturbed ISMF (Funsten et al. 2009; McComas et al. 2013; Funsten et al. 2013). We use the RC location given by Funsten et al. (2009) and McComas et al. (2009) (see

Table 1). The recent energy averaged RC position (Funsten et al. 2013) differs from it only by $1.6^{\circ}$. A change by this amount would not affect our results significantly. 
There are two crucial ingredients of our explanation. First, the heliolatitudes of V1 and the undisturbed ISMF happen to be approximately the same ( $34.5^{\circ}$, see Table 1), with small angular distance between the two directions $\left(\sim 27^{\circ}\right)$. Second, for a strong interstellar field, the draping around the HP has a simple structure, the evolution of which (in the region of HP not far from the undisturbed field direction) can be traced for the field magnitude decreasing towards the realistic values.

The essence of our argument is presented in Figure 1. The V1 and ISMF (RC) directions are shown on a sky map in orthogonal projection and Sun-centered equatorial (heliographic inertial) coordinate 1 . From V1 observations we know (Burlaga, Ness \& Stone 2013) that the magnetic field on the inner side of the HP is very close to the Parker spiral, that is to the $-\mathrm{T}$ direction in the RTN system2. Projected on the sky map, the solar magnetic field line is tangent to the heliographic parallel passing through the V1 position. $\mathrm{B}_{\mathrm{IN}}$ and $\mathrm{B}_{\mathrm{OUT}}$ denote the directions of the magnetic field measured by $\mathrm{V} 1$ before and after the crossing the HP, respectively.

The projection of the ISMF line passing through V1 position must link this point with the direction of the unperturbed ISMF (RC). If the draping pattern is such that the projected ISMF line reaches RC from V1 with little change in direction, the ISMF at V1 must be approximately parallel to the inner field simply because $\mathrm{V} 1$ and $\mathrm{RC}$ have the same heliolatitude.

\footnotetext{
1 http://omniweb.gsfc.nasa.gov/coho/helios/coor_des.html

2 http://www.srl.caltech.edu/ACE/ASC/coordinate_systems.html. The transformation from heliographic inertial to RTN coordinates follows from Franz \& Harper (2002).
} 


\section{Simple Draping Model}

The simplest ('idealized') draping model with this property is illustrated in Figure 1, where the selected draped ISMF field lines are shown as thick blue lines. We chose one of them to pass through the V1 and another through V2 position. In this model, based on

the well-known solution by Parker (Parker 1961) (see also Figure 21), the projected lines are arcs of great circles and originate in the undisturbed ISMF direction (RC).

In Parker's solution the star is at rest relative to the interstellar medium with insignificant plasma density and strong magnetic field. The stellar wind plasma flows away from the star, forming two streams, parallel and anti-parallel to the ISMF direction. Below we shall show, from our 3DMHD simulations (Czechowski et al. 2013), that the same idealized draping model can be regarded as the high ISMF strength limit for the case of the Sun, which is moving through the medium including the magnetized plasma. Moreover, when the ISMF strength is decreased to values actually observed by V1 beyond the HP (4-6 $\mu \mathrm{G}$ ) (Burlaga et al. 2013), or, since the draped field near the HP can be stronger than the undisturbed ISMF (by $\sim 40 \%$ if the field is as weak as $\sim 3 \mu \mathrm{G}$ ), even to lower values (3 $\mu \mathrm{G}$ or $2.7 \mu \mathrm{G}$, Grygorczuk et al. (2011); Heerikhuisen et al. (2014); BenJaffel et al. (2013)), the draped field near V1 direction in our heliographic projection remains similar enough in structure to the idealized model (Fig. 3) to agree approximately with V1 observations. The red line in Figure 1 shows the draped field line obtained for the ISMF of $4 \mu \mathrm{G}$. The angle between the red line and the heliographic parallel passing through V1 direction is larger than for the idealized draping model (the blue line). However, this is consistent with V1 observations, which correspond to the small but nonzero angle between the field outside and inside HP.

To agree with the V1 measurements, the red line at V1 position should be strictly tangent not to the heliographic parallel, but to the (V1, $\mathrm{B}_{\mathrm{OUT}}$ ) plane (defined by the V1 
direction and the direction of the magnetic field measured by V1 outside the HP). In our projection this plane is represented by the great circle passing through the points V1 and $\mathrm{B}_{\text {OUT }}$. Figure 1 shows that the draped field line corresponding to our draping model for the ISMF strength of $4 \mu \mathrm{G}$ is indeed close to being tangent to this great circle. Moreover, the draped field direction in space derived from the model $\mathrm{B}_{m h d}^{V 1}$ (the red square) is close $\left(\sim 10^{\circ}\right)$ to the measured field direction $\mathrm{B}_{\text {OUT }}$ (the black square).

Figure 1 1also shows the heliographic parallel and the magnetic field line for the idealized draping model, passing through the V2 position. The angle between the field line and the parallel is clearly large $\left(30^{\circ}\right)$ implying that the solar magnetic field and the draped ISMF will not be close in direction along the V2 trajectory. Similar values $\left(\sim 32^{\circ}\right.$ and $\left.31^{\circ}\right)$ are obtained in our 3DMHD simulations for the ISMF strength of 4 and $3 \mu \mathrm{G}$, respectively. The angular distance between V2 position and the $\mathrm{RC}$ is also much larger $\left(\sim 97^{\circ}\right)$ than for V1.

The vicinity of the V1 direction is not the only region where the magnetic fields outside and inside the HP can be parallel (or antiparallel) to each other. In the idealized draping model, each of the interstellar magnetic field lines become tangent to a heliographic parallel at two points. The dashed blue line in Figure 1 shows a locus of such points situated between the north pole and ISMF directions (a similar line appears in the southern hemisphere). The draped field line which crosses this oval becomes tangent to the heliographic parallel at the crossing point (as illustrated by the elongated blue line in the north). If a spacecraft trajectory direction would be close to this line, then, assuming the idealized draping model, the solar and the interstellar fields observed at the heliopause would be approximately parallel (or antiparallel, for opposite polarity of the solar field). The actual direction of Voyager 1 trajectory (at the same solar latitude and close in longitude to the $\mathrm{RC}$ ) satisfies this condition. 


\section{MHD Models}

3DMHD simulations for the case of a star moving through a strongly magnetized (up to $20 \mu \mathrm{G}$ ) interstellar medium including also the plasma and neutral gas components (Czechowski et al. 2013) led to a two-stream structure of the stellar plasma flow. In this case the streams (containing most of the plasma flow) are slightly deflected from the interstellar field direction. The rest of the outflowing stellar plasma forms a tail opposite to the stellar motion. The two stream structure becomes less pronounced for larger values of the neutral interstellar hydrogen density. Nevertheless, the draping structure for the strong interstellar field case (Fig. 3, black arcs) remains similar to our simple draping model (Fig. 1, blue lines).

After the crossing of the HP, V1 is observing the interstellar field strength between 4 and $6 \mu \mathrm{G}$. To check the draping structure for the ISMF in this range we performed 3DMHD simulation for the local field strengths of $6,5,4,3$, and $2.7 \mu \mathrm{G}$, with the asymptotic field directed towards the IBEX RC. We found that the weaker the field the more its draping structure differs from the one of the strong $(20 \mu \mathrm{G})$ field case. Figure 3 illustrates this difference for the ISMF strength of $3 \mu \mathrm{G}$. Although the draped field is not as simple as for the $20 \mu \mathrm{G}$, in our region of interest (between the undisturbed field (RC) and V1 direction) its structure simplifies and takes the form consistent with V1 magnetic field measurements. As explained above, the draped magnetic field line passing through the V1 trajectory is close to tangent to the plane defined by the $\mathrm{V} 1$ and $\mathrm{B}_{\mathrm{OUT}}$ directions, and close in direction to the solar magnetic field on the inner side of the HP.

The direction of the draped magnetic field in space at the V1 position following from our models depends on the assumed ISMF strength. For a very strong field $(20 \mu \mathrm{G})$ it is different from the $\mathrm{V} 1$ result $\mathrm{B}_{\mathrm{OUT}}$, but for the weaker and more realistic ISMF strength values it becomes close to $\mathrm{B}_{\text {OUT }}$ (within about $10^{\circ}$ ). At the same time, the angle between 
the draped magnetic field at V1 and the heliographic parallel increases from a low value (less than $10^{\circ}$ ) obtained for the idealized draping model and for strong ISMF $(20 \mu \mathrm{G})$ to a somewhat higher value $\left(15^{\circ}\right.$, close to V1 observations) for the realistic ISMF.

In our calculations we use 3DMHD time-stationary numerical code describing the interaction of the solar wind with the LISM (boundary conditions for our models are shown in Table 2). The parameters of the solar wind are based on Ulysses data (Ebert et al. 2009). The interstellar medium parameters were chosen to agree with observations from the IBEX and Voyager 1 spacecraft (Gurnett et al. 2013), and model estimations. The code employs a fixed grid. A uniform flux of neutral hydrogen is assumed (Bzowski et al. 2009; Ratkiewicz \& Grygorczuk 2008). The solar magnetic field is disregarded to limit the undesired numerical effects (like artificial diffusion of the magnetic field at the HP) that could affect the heliopause region. In effect we assume that, as in ideal MHD case, there is no coupling between the magnetic fields across the HP.

We use the models to obtain the shape of the HP and the structure of the draped magnetic field. Since the HP in our models is not a discontinuity but a transition of finite width, we cannot reliably calculate the behaviour of quantities like the magnetic field strength or plasma density with distance in vicinity of the HP. For the magnetic field direction we find that the agreement of the $4 \mu \mathrm{G}$ and $3 \mu \mathrm{G}$ cases with $\mathrm{V} 1$ observations is satisfactory. The calculated ISMF direction at V1 just outside the HP (the red squares marked $\mathrm{B}_{\mathrm{mhd}}^{V 1}$ in Figure 1 and Figure 3) are deflected by $\sim 11^{\circ}$ from the observed direction. While the distance to the HP is overestimated, the obtained termination shock distances at V1 and V2 are within few percent from the measured ones (Stone et al. 2005, 2008) when the slow wind speed is taken to be $500 \mathrm{~km} / \mathrm{s}$, but smaller (by less than $20 \%$ for the slow wind speed of $400 \mathrm{~km} / \mathrm{s}$. 


\section{Discussion and Conclusions}

Throughout this paper we are using the heliographic projection. We are interested in the magnetic field lines near the heliopause. The inner and the outer magnetic fields are then draped over the same surface. Since the heliopause is not strictly perpendicular to the radial direction, the angles between the projected field lines are not the same as the angles between the field lines in space. However, as long as we exclude the case of a heliopause tangent to the radial direction, the lines draped over the same element of the heliopause and strictly parallel (antiparallel) in projection have the same property also in space. We can therefore restrict attention to the heliographic projection and do not have to specify the shape of the actual heliopause. If the considered part of the heliopause is not very far from being perpendicular to radial direction, the field lines forming a small angle in projection, will also form a small angle in space.

Our results clarify the cause of similar magnetic field directions on both sides of the $\mathrm{HP}$, as observed by V1: the heliographic latitude of V1 happens to be the same as that of the undisturbed ISMF (to be given by the IBEX RC). We have shown that for the wide range of the ISMF strength of values, including those actually observed by V1 beyond the HP, the draped interstellar field near V1 trajectory (calculated with zero heliospheric magnetic field to avoid artificial field diffusion across the boundary) must then be close in direction to the solar inner heliosheath magnetic field. For realistic ISMF strength values, our model results obtained for the draped field direction in space are within $10^{\circ}$ from V1 observations beyond the HP. For Voyager 2 we predict that the difference between the inner and the outer magnetic field directions at the heliopause will be significantly larger than the one observed by Voyager $1\left(\sim 30^{\circ}\right.$ instead of $\left.\sim 20^{\circ}\right)$ and that the outer field direction will be close $\left(7^{\circ}-9^{\circ}\right.$ off $)$ to the $\mathrm{RC}$.

The explanation is natural and simple, and removes the only objection against the 
claim that V1 reached the interstellar medium. It supports the interpretation of the ribbon center as the direction of the undisturbed ISMF, with the ISMF oriented towards the northern hemisphere It is consistent with the classical picture of the HP not dominated by the turbulence or reconnection effects. The reasonable agreement between V1 and our results for the magnetic field direction gives us confidence that the results are not affected by the approximations inherent to our models.

Approximate agreement with V1 magnetic field measurements was also achieved in numerical models that differ significantly from our approach. Borovikov \& Pogorelov (2014) (see also Pogorelov et al. (2009)), who take HP instability into account, obtain a solution in which V1 is moving through a local interstellar plasma intrusion, partly surrounded by the heliosheath plasma. They predict that V1 may re-enter the heliosheath in the near future. Opher \& Drake (2013), from numerical calculations that include both solar and interstellar magnetic fields, conclude that the presence of solar magnetic field strongly affects the draping of the ISMF over the HP. In their solution, for different asumptions about the undisturbed ISMF, the draped ISMF is close in direction to the heliospheric magnetic field in a large area of the HP including also the V1 and V2 trajectory directions. Calculations of Borovikov \& Pogorelov (2014) do not confirm this result. The solar field assumed by Opher \& Drake $(2013)$ is very simplified. In particular, the sector polarity structure is ignored. Contrary to Opher \& Drake (2013), we assume that the solar magnetic field does not strongly affect the shape of the HP and the draping pattern. The similarity of the field directions on both sides of the HP follows from the relative directions of V1 and the undisturbed ISMF.

This work was supported by the Polish National Science Center grant 2012-06-M-ST900455. 


\section{REFERENCES}

BenJaffel, L., Strumik, M., Ratkiewicz, R. \& Grygorczuk, J. 2013, ApJ, 779, 130

Borovikov, S. N., \& Pogorelov, N. V. 2014, ApJ, 783, L16

Burlaga, L. F., Ness, N. F., \& Stone, E.C. 2013, Science, 341, 147

Burlaga, L. F., Ness, N. F., Gurnett, D. A., \& Kurth, W. S. 2013, ApJ, 778, L13

Bzowski, M., Moebius, E., Tarnopolski, S., Izmodenov, V., Gloeckler, G. 2009, Space Sci. Rev., 143, 177

Czechowski, A., Grygorczuk, J., McComas, D., \& Ratkiewicz, R. 2013, A\&A, submitted

Ebert, R. W., McComas, D. J., Elliott, H. A., Forsyth, R. J., \& Gosling, J. T. 2009, J. Geophys. Res., 114, 1109

Fisk, L. A. \& Gloeckler, G. 2013, ApJ, 776, 79

Florinski V. 2013, arXiv:1302.2179v1

Franz, M., \& Harper, D. 2003, Planetary \& Space Science, 50, 217-233

Funsten, H. O., Allegrini, F., Crew, G. B., DeMajistre, R., Frisch, P. C., et al. 2009, Science, 326,964

Funsten, H. O., De Majistre, R., Frisch, P. C., Heerikhuisen, J., Higdon, D. M., Janzen, P., Larsen, B. A., Livadiotis, G., McComas, D. J., Moebius, E., Reese, C. S., Reisenfeld, D. B., Schwadron, N. A., \& Zirnstein, E. J. 2013, ApJ, 776, 30

Grygorczuk, J., Ratkiewicz, R., Strumik, M. \& Grzedzielski, S. 2011, ApJ, 727, L48

Gurnett, D. A., Kurth, W. S., Burlaga, L. F., and Ness, N. F. 2013, ApJ, 341, 1492 
Heerikhuisen, J., Zirnstein, E. J., Funsten, H. O., Pogorelov, N. V., \& Zank, G. P. 2014, ApJ, 784, 73

McComas, D. J. \& Schwadron, N. A. 2013, ApJ, 758, 19

McComas D. J.,Allegrini, F., Bochsler, P., Bzowski, M., Christian, E. R., et al. 2009, Science, 326,13

McComas, D.J., Dayeh,M.A., Funsten, H. O., Livadiotis, G., \& Schwadron, N. A. 2013, ApJ, 771, 77

Opher, M., \& Drake, J. F. 2013, ApJ, 778, L26

Parker, E. N. 1961, ApJ, 134, 20-27

Pogorelov, N. V., Borovikov, S. N., Zank, G. P., \& Ogino, T. 2009, ApJ, 696, 1478-1490

Ratkiewicz, R., \& Grygorczuk, J. 2008, Geophys. Res. Lett., 35, L23105

Schwadron, N. A. \& McComas, D. J. 2013, ApJ, 778, L33 9

Stone, E. C., Clummings, A. C., McDonald, F. B., Heikkila, B. C., Lal, N., \& Webber, W. R. 2005, Science, 309, 2017

Stone, E. C., Clummings, A. C., McDonald, F. B., Heikkila, B. C., Lal, N., \& Webber, W. R. 2008, Nature, 454, 71

Stone, E. C., Cummings, A. C., McDonald, F. B., Heikkila, B. C., Lal, N., \& Webber, W. R. 2013, Science, 341, 150 


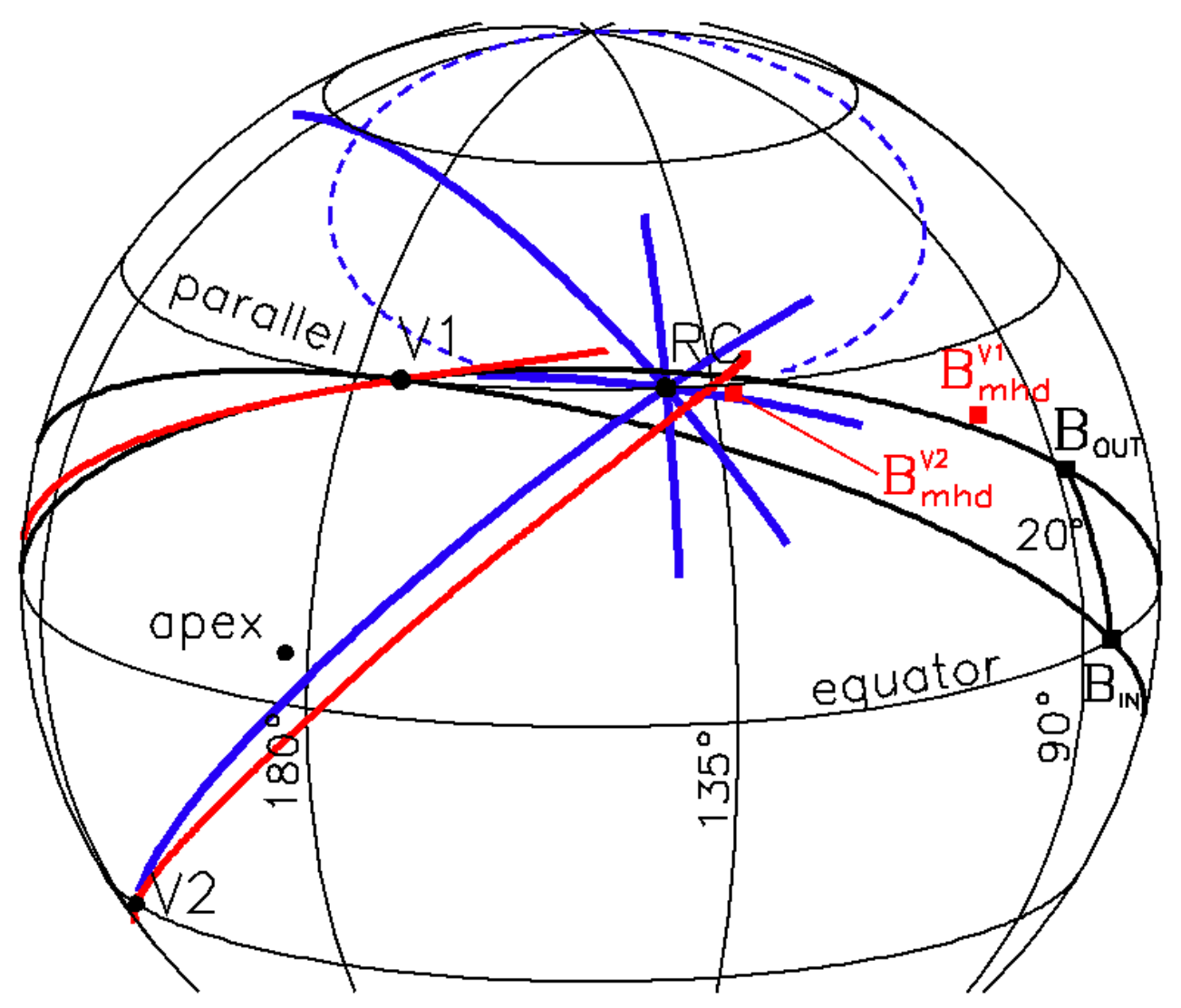

Fig. 1.- Sky map in orthogonal projection and heliographic inertial coordinates. Shown are: Voyager 1 (V1), Voyager $2(\mathrm{~V} 2)$, IBEX ribbon center (RC), interstellar inflow direction (apex), magnetic field directions measured by V1 before and after crossing the heliopause $\left(\mathrm{B}_{I N}\right.$ and $\mathrm{B}_{\text {OUT }}$, respectively). Two great circles (black) link $\mathrm{V} 1$ with $\mathrm{B}_{\text {OUT }}$ and $\mathrm{B}_{\mathrm{IN}}$ (the latter is tangent to the heliographic parallel at V1). Thick blue lines are the interstellar magnetic field lines in the idealized draping model. The dashed blue oval is a locus of points where the idealized draped interstellar field lines become tangent to the local heliographic parallel (illustrated by the extended field line in the north). The thick red lines are the draped magnetic field lines obtained from our simulation for $4 \mu \mathrm{G}$ case. The red squares $\left(\mathrm{B}_{m h d}^{V 1}\right.$ and $\left.\mathrm{B}_{m h d}^{V 2}\right)$ are the directions of the interstellar field just outside the heliopause at directions of V1 and V2 for the same simulation. 


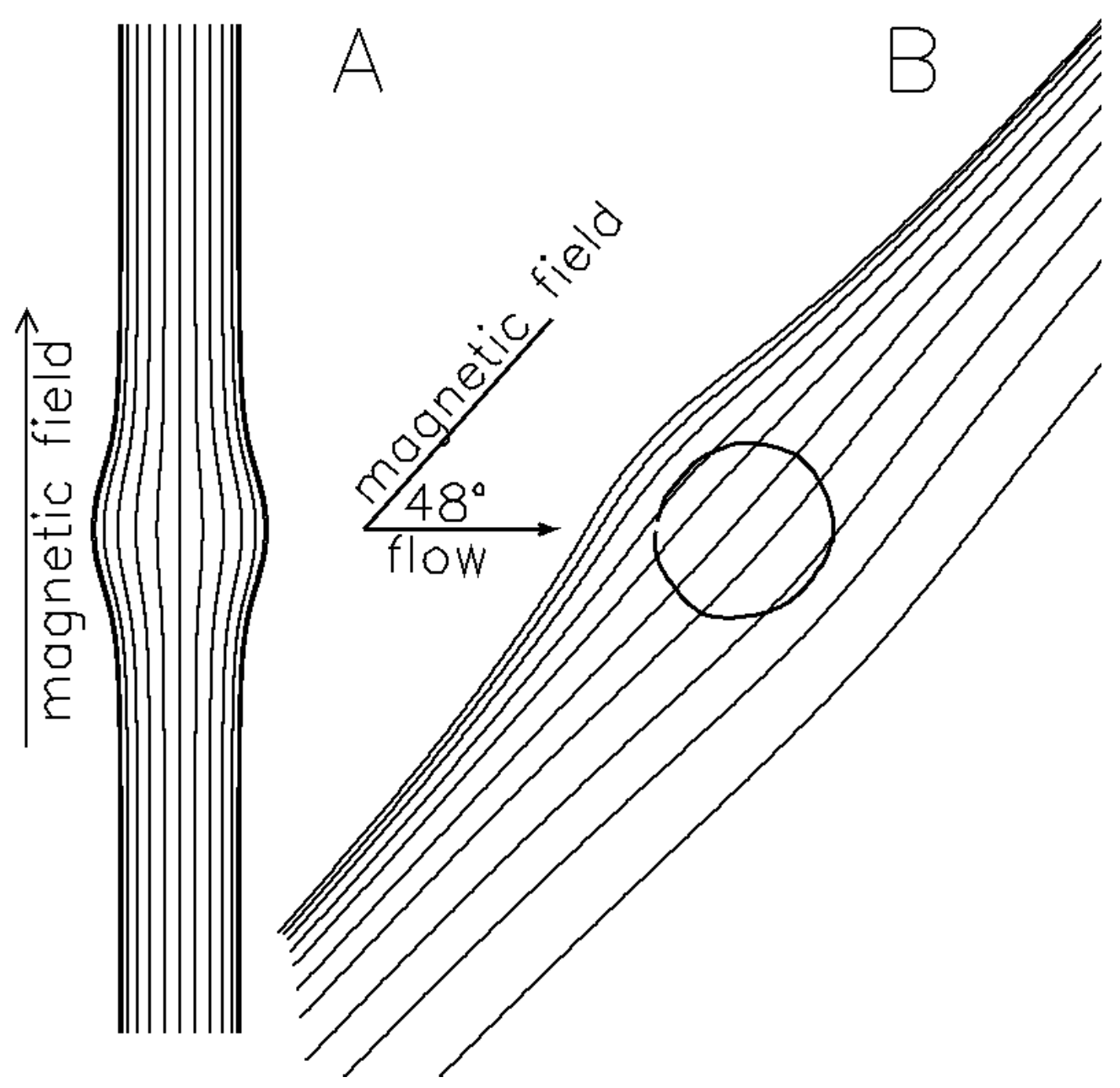

Fig. 2.-Draped interstellar magnetic field lines: A) in Parker's solution (star at rest relative to the interstellar medium with insignificant plasma density), and B) obtained from 3DMHD simulations for spherically-symmetric solar wind and interstellar magnetic field of $20 \mu \mathrm{G}$, inclined $48^{\circ}$ to interstellar plasma flow (projection on a plane which contains interstellar flow and field vectors). 


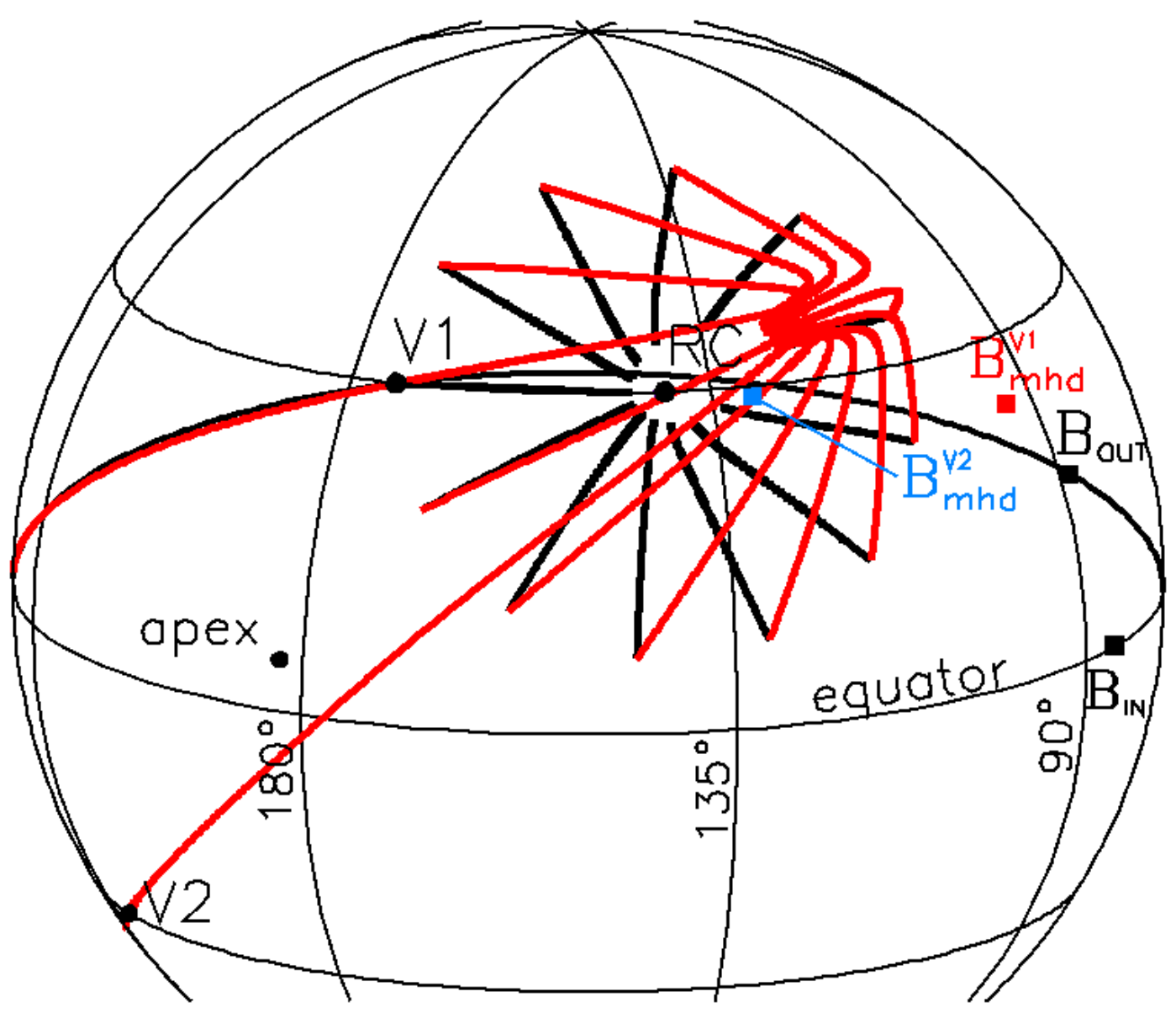

Fig. 3.- Comparison of draped interstellar magnetic field lines from our simulations for field strengths of 20 and $3 \mu \mathrm{G}$ (black and red, respectively). The lines originate from selected points just outside the heliopause, distributed over a circle of radius $27^{\circ}$ with the same center as the IBEX ribbon. 
Table 1: Characteristic directions in galactic (GAL), solar ecliptic (SE), solar equatorial $\left(\mathrm{HGI}^{\mathrm{a}}\right)$, and $\mathrm{RTN}^{\mathrm{b}}$ coordinates $(\mathrm{J} 2000)$.

\begin{tabular}{|c|c|c|c|c|c|c|c|c|}
\hline & \multicolumn{2}{|c|}{ GAL } & \multicolumn{2}{|c|}{$\mathrm{SE}$} & \multicolumn{2}{|c|}{$\mathrm{HGI}^{\mathrm{a}}$} & \multicolumn{2}{|c|}{$\mathrm{RTN}^{\mathrm{b}}$} \\
\hline & lon & lat & lon & lat & lon & lat & lon & lat \\
\hline & (deg) & (deg) & (deg) & (deg) & (deg) & (deg) & (deg) & $(\operatorname{deg})$ \\
\hline V1 & 32.7 & 28.1 & 254.9 & 35.0 & 174.1 & 34.5 & & \\
\hline $\mathrm{V} 2$ & 342.1 & -31.2 & 289.4 & -34.2 & 217.2 & -30.0 & & \\
\hline $\mathrm{RC}\left(=\mathrm{B}_{\mathrm{IS}}\right)$ & 32.9 & 55.3 & 221.0 & 39.0 & 140.9 & 34.6 & & \\
\hline apex & 5.3 & 12.0 & 259.0 & 4.98 & 182.6 & 5.4 & & \\
\hline $\mathrm{B}_{\mathrm{IN}}$ & 232.0 & 60.4 & 159.6 & 7.2 & 83.9 & 0.0 & 270 . & 0. \\
\hline $\mathrm{B}_{\mathrm{OUT}}$ & 197.2 & 75.1 & 163.7 & 26.0 & 88.0 & 18.8 & 284. & 13. \\
\hline$B_{\mathrm{MHD}}^{\mathrm{V} 1} \quad 2.7 \mu \mathrm{G}^{1}$ & 155. & 80. & 169. & 35. & 93. & 28. & 293. & 18. \\
\hline$B_{\mathrm{MHD}}^{\mathrm{V} 1} \quad 3 \mu \mathrm{G}^{1}$ & 164. & 73. & 161. & 35. & 95. & 28. & 295 . & 17. \\
\hline$B_{\mathrm{MHD}}^{\mathrm{V} 1} 4 \mu \mathrm{G}^{1}$ & 168. & 53. & 137. & 34. & 100. & 27. & 298. & 13. \\
\hline$B_{\mathrm{MHD}}^{\mathrm{V} 1} \quad 20.0 \mu \mathrm{G}$ & 28. & 63. & 211. & 36. & 132. & 31. & 325. & 3. \\
\hline$B_{\mathrm{MHD}}^{\mathrm{V} 1} \quad 3 \mu \mathrm{G}^{2}$ & 159. & 71. & 159. & 37. & 93. & 30. & 294. & 19. \\
\hline$B_{\mathrm{MHD}}^{\mathrm{V} 1} 4 \mu \mathrm{G}^{2}$ & 126. & 83. & 176. & 36. & 99. & 29. & 299. & 16. \\
\hline $\mathrm{B}_{\mathrm{MHD}}^{\mathrm{V} 2} \quad 2.7 \mu \mathrm{G}^{1}$ & 36. & 65. & 208. & 39. & 129. & 34. & 253. & 29. \\
\hline $\mathrm{B}_{\mathrm{MHD}}^{\mathrm{V} 2} \quad 3 \mu \mathrm{G}^{1}$ & 35 & 64. & 209. & 39 . & 130 . & 33. & 254 & 30. \\
\hline $\mathrm{B}_{\mathrm{MHD}}^{\mathrm{V} 2} \quad 4 \mu \mathrm{G}^{1}$ & 34 & 62. & 212 . & 39 . & 132. & 34 & 256 . & 31. \\
\hline$B_{\mathrm{MHD}}^{\mathrm{V} 2} \quad 20.0 \mu \mathrm{G}$ & 30 & 53. & 224 & 37. & 143. & 33. & 265. & 36. \\
\hline $\mathrm{B}_{\mathrm{MHD}}^{\mathrm{V} 2} \quad 3 \mu \mathrm{G}^{2}$ & 37. & 62. & 212 . & 40 & 133. & 35. & 255. & 32. \\
\hline $\mathrm{B}_{\mathrm{MHD}}^{\mathrm{V} 2} \quad 4 \mu \mathrm{G}^{2}$ & 35. & 60 & 215. & 40 & 135. & 35. & 257. & 33. \\
\hline
\end{tabular}

${ }^{a}$ http://omniweb.gsfc.nasa.gov/coho/helios/coor_des.html

${ }^{b}$ http://www.srl.caltech.edu/ACE/ASC/coordinate_systems.html

1 Table 2 solar wind case 400/750.

2 Table 2 solar wind case 500/750. 
Table 2: Boundary conditions for MHD calculations ${ }^{1}$

\begin{tabular}{ccccc|cc}
\hline \hline \multicolumn{5}{c|}{ LISM parameters } & \multicolumn{2}{c}{ Solar Wind parameters ${ }^{2}$} \\
\hline $\mathrm{B}_{\mathrm{IS}}$ & $\mathrm{T}_{\mathrm{IS}}$ & $\mathrm{n}_{\mathrm{IS}}$ & $\mathrm{n}_{\mathrm{H}}$ & $\mathrm{V}_{\mathrm{IS}}$ & $\mathrm{V}_{\mathrm{SW}, 1 \mathrm{AU}}$ & $\mathrm{n}_{\mathrm{SW}, 1 \mathrm{AU}}$ \\
\hline$(\mu \mathrm{G})$ & $(\mathrm{K})$ & $\left(\mathrm{cm}^{-3}\right)$ & $\left(\mathrm{cm}^{-3}\right)$ & $(\mathrm{km} / \mathrm{s})$ & $(\mathrm{km} / \mathrm{s})$ & $\begin{array}{c}\left(\mathrm{cm}^{-3}\right) \\
\text { slow/fast }\end{array}$ \\
\hline & & & & & slow/fast & slow \\
\hline 20 & 6300 & 0.04 & 0.1 & 23.2 & 750 & 4.2 \\
$4,5,6$ & 6300 & 0.06 & 0.1 & 23.2 & 400 & 5.55 \\
$2.7,3,4,5$ & 6300 & 0.06 & 0.1 & 23.2 & $400 / 750$ & $5.55 / 1.58$ \\
3,4 & 6300 & 0.06 & 0.1 & 23.2 & $500 / 750$ & $5.55 / 2.46$ \\
\hline
\end{tabular}

1 The inner boundary of the calculation region is at $15 \mathrm{AU}$, the outer at $5849 \mathrm{AU}$ from the Sun.

${ }^{2}$ In the slow/fast solar wind cases, the slow wind flows within $36^{\circ}$ from the solar equator. 